\title{
Distrattori e Chiavi in un Cloze Lessicale a Scelta Multipla di Livello Avanzato: L'Opportunità di Considerare il Giudizio di NATIVI EsPERTI
}

\author{
Paolo Torresan*
}

ABSTRACT: In questa nostra indagine abbiamo somministrato 2 cloze lessicali a scelta multipla, destinati a studenti di livello $\mathrm{C} 2$ e tratti dalla certificazione di italiano come lingua straniera CILS (Università per Stranieri di Siena), a due gruppi diversi di nativi esperti $(\mathrm{n}=37)$, e abbiamo analizzato le risposte divergenti rispetto a quelle previste dal certificatore. Dall'analisi emerge la plausibilità di alcuni distrattori. Le conclusioni cui giungiamo riguardano la necessità, da parte del certificatore, di ricorrere al giudizio di nativi esperti come forma di validazione del test.

PAROLE CHIAVE: test; validità; cloze; lessico.

RESUMO: Na pesquisa aqui apresentada, dois grupos diferentes de falantes nativos com elevado grau de conhecimento da língua $(n=37)$ realizaram 2 cloze lexicais de múltipla escolha destinados a estudantes de nível C2 e extraídos do exame de certificação do italiano como língua estrangeira CILS (Università per Stranieri di Siena). As respostas divergentes em relação às previstas pelos responsáveis pela certificação foram analisadas. Da análise emerge a plausibilidade de alguns distratores. Nas conclusões discutimos a necessidade de os responsáveis pela certificação considerar o julgamento de falantes nativos com alto grau de conhecimento linguístico como forma de validar o teste. 
PALAVRAS-CHAVE: teste; validade; cloze; léxico.

ABSTRACT: For the purpose of this study, we had two different groups of native experts ( $n=37)$ sit 2 multiple choice reading cloze tests intended for $C 2$ level students taken from the CILS certification in Italian as a foreign language (Università per Stranieri, Siena). We then analyzed the answers not coinciding with those intended by the certifying body. The analysis demonstrates the presence of certain plausible distractors. We discuss certain conclusions regarding the certifier's need to take the judgment of native experts into account as a means of validating the test.

KEYWORDS: assessment; test validity; cloze; lexical competence. 


\section{Il cloze lessicale a scelta multipla ${ }^{1}$}

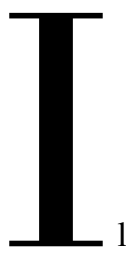

1 candidato di un cloze lessicale a scelta multipla (d'ora in poi CSM) deve scegliere, durante l'attività di completamento, la voce più adatta all'interno di una rosa di termini che hanno una relazione semantica tra loro (sinonimi; iperonimo/iponimi; rapporti di connotazione/ denotazione; ecc.) e questa prova viene considerata cognitivamente meno ardua rispetto a un cloze libero (CAI, 2012). Ciò che viene richiesto di fare a un apprendente di livello C2 (Quadro Comune Europeo di Riferimento per le Lingue - QCER, Consiglio d'Europa, 2002) che si appresti a eseguire questo esercizio è di dar prova di un uso della lingua sensibile alle sfumature socioculturali e stilistiche, visto che un apprendente di livello C2: 
possiede un repertorio lessicale molto ampio comprendente anche espressioni idiomatiche e colloquiali e dimostra di saper utilizzare le parole in modo adeguato, pertinente e preciso. È capace di produrre sinonimi in registri linguistici differenti e identificare la connotazione sociale delle parole ai fini di una loro adeguata realizzazione (BARNI et al., 2009, p. 40).

L'oggetto della nostra indagine sono CSM di livello C2, tratti dalla certificazione CILS:

- sessione estiva 2009 [cloze A]

- sessione estiva 2012 [cloze $\mathrm{B}]$.

\section{Il campione di studio}

I nativi variano tra di loro in termini di competenza circa la L1: non tutti possiedono la stessa padronanza del codice. In merito alla conoscenza lessicale, la variabilità individuale è sensibile:

- al grado di istruzione (una persona con bassa scolarità ha un lessico più povero rispetto ad una persona istruita);

- all'età (un adolescente ha un lessico più ridotto rispetto ad un adulto);

- al tipo di professione e di hobby (se l'una e l'altro, cioè, comportano un confronto pressoché quotidiano con la L1, l'individuo consolida un lessico ampio) $)^{2}$.

Un madrelingua a cui venga sottoposto un test di livello $\mathrm{C} 2$, come la prova lessicale di cui parliamo, non è detto, quindi, che lo possa portare a termine con esito. Se ha una bassa scolarità, se è molto giovane o non utilizza quotidianamente la lingua scritta in un contesto professionale, non è detto che riesca a far meglio di uno studente straniero competente e con un buon profilo intellettuale (che il livello avanzato e quello della padronanza richiedono).

2 Cfr. sulle tre variabili Hulstijn, 2011. 
Al contrario, un nativo colto/esperto (d'ora in poi NE) può portare a compimento un compito tarato su misura per un apprendente straniero di livello avanzato; il suo giudizio può anzi - ed è questa la nostra ipotesi - assumere un carattere regolativo per l'item writer, sia nella fase di confezione, che in quella di calibrazione e infine durante la validazione della prova (cfr. MULDER e HULSTIJN, 2011) $)^{3}$.

In questo studio abbiamo sottoposto i due CSM tratti dalla certificazione CILS a due gruppi omogenei di NE $(n=37)$ : cittadini italiani in possesso di un titolo di laurea, di età superiore ai trent'anni, che usano l'italiano per la redazione pressoché quotidiana di testi all'interno della loro attività professionale (impiegati, ricercatori, liberi professionisti). Il loro giudizio, si vedrà, ci impone di riflettere sull'adeguatezza delle opzioni.

\subsection{Le prove}

Le prove oggetto di studio hanno le seguenti caratteristiche in comune:

- il livello: $\mathrm{C} 2$

- il tipo di fonte: un testo giornalistico

- l'argomento: l'evoluzione della lingua italiana

- la struttura: 15 item con 4 distrattori ciascuno

- la trasformazione: il certificatore ha operato delle modifiche su una fonte tratta dall'attualità.

Riguardo a quest'ultimo punto, nelle Appendici 1 e 2 il lettore può accedere a un prospetto con evidenziate, per ciascuna delle prove, le modifiche incorse sul testo originale (d'ora in poi TO). In entrambi i casi, il testo che ne è derivato (TM, testo modificato) presenta criticità che tuttavia, presumiamo, non influiscono sulla performance del candidato.

3 La confezione di un CSM può prevedere, del resto, ulteriori parametri esterni di confronto, quali il giudizio di un lessicografo e il ricorso a corpora. 


\subsection{Analisi delle risposte dei madrelingua}

Entrambi i CSM sono stati somministrati a due gruppi distinti di NE (n=37). Il risultato della somministrazione è discusso nei paragrafi che seguono.

\subsubsection{Cloze A}

Di seguito presentiamo testo e item del cloze A (C2, sessione estiva 2009).

\section{Completa. Come nell'esempio (0).}

\section{Grammatica: Viene Avanti Uno Strano Italiano. Colpa Di Internet E Tv? No, Nostra}

Come parliamo? Come sta l'italiano oggi? Come sta cambiando? È vero che chi parla male pensa male e vive male, come diceva Nanni Moretti? L'Università Ca' Foscari di Venezia lancia dati (0) allarmanti : all'ultimo esame di ammissione alla Facoltà di Lettere il 44 per cento dei candidati sarebbero stati bocciati per (1) ortografici.

Ma i linguisti ridimensionano l' (2) e alcuni luoghi comuni, come quello che vede in internet, tv e inglese il male (3) "La lingua italiana e la sua grammatica stanno benone" afferma il professor Tullio De Mauro, ex Ministro dell'Istruzione: "Mai nei secoli erano state adoperate da un numero così ampio di (4) e mai tanto studiate da stranieri che apprendono l'italiano come seconda lingua. Siamo noi che stiamo meno bene: secondo una (5) soltanto un terzo della popolazione ha gli (6) culturali sufficienti a servirsi con scioltezza e correttezza di una lingua piena di (7) come l'italiano".

Negli ultimi dieci anni, secondo una ricerca Treccani, i giornali hanno introdotto o in qualche modo lanciato $4.163(8)$ nuovi: da gossipare ad attapirarsi, da botulinata a fannullonismo. "Una volta l'informazione parlava meglio" sostiene Andrea De Benedetti, giornalista e linguista. C'era più (9) facevano corsi di dizione. Ora la provenienza geografica nell' (10) è quasi ostentata. Il linguaggio era più didascalico, anche perché (11) ad un pubblico meno alfabetizzato, mentre ora è diventato più (12) e meno creativo. Si abusa di slogan o frasi fatte: valzer delle poltrone, guerra dei numeri... Scorciatoie comunicative che diventano subito modelli perdendo il (13) metaforico. C'è più sciatteria”. Stefano Bartezzaghi, direttore del recente Festival della Linguistica a Poggibonsi (Siena), cerca l'(14) positivo:

"L'italiano viene calpestato di più perché si parla di più, e questo non è un (15) negativo. Più auto circolano e più incidenti, statisticamente, si contano". 


\begin{tabular}{lllll}
\hline 0. & A) allarmanti & B) dolorosi & C) angosciosi & D) pericolanti \\
\hline 1. & A) equivoci & B) difetti & C) errori & D) peccati \\
\hline 2. & A) emergenza & B) imprevisto & C) eventualità & D) urgenza \\
\hline 3. & A) illimitato & B) completo & C) incondizionato & D) assoluto \\
\hline 4. & A) oratori & B) parlanti & C) parolai & D) relatori \\
\hline 5. & A) perizia & B) considerazione & C) stima & D) opinione \\
\hline 6. & A) strumenti & B) arnesi & C) attrezzi & D) apparecchi \\
\hline 7. & A) diversità & B) alterazioni & C) conversioni & D) varianti \\
\hline 8. & A) vocaboli & B) argomenti & C) segni & D) significati \\
\hline 9. & A) concentrazione & B) riflessione & C) attenzione & D) diligenza \\
\hline 10. & A) espressione & B) atteggiamento & C) impostazione & D) esibizione \\
\hline 11. & A) dedicato & B) rivolto & C) concentrato & D) rappresentativo \\
\hline 12. & A) ripetitivo & B) regolare & C) normale & D) ordinato \\
\hline 13. & A) merito & B) rilievo & C) pregio & D) valore \\
\hline 14. & A) aspetto & B) esponente & C) ingrediente & D) elemento \\
\hline 15. & A) episodio & B) fenomeno & C) affare & D) problema \\
\hline
\end{tabular}

Nella tabella che segue si riporta il profilo delle risposte dei soli candidati $(n=26)$ che hanno commesso uno o più errori, stando alla logica dell'item writer. 


\section{LEGENDA}

in grigio sono riportate le chiavi

$\mathrm{x}$ : errori

per ogni singolo distrattore

in rosso sono riportate le risposte divergenti da parte dei $\mathrm{NE}$

tot: errori per ogni singolo item

\begin{tabular}{|c|c|c|c|c|c|c|c|c|c|c|c|c|c|c|c|c|c|c|c|c|c|c|c|c|c|c|c|c|c|}
\hline & & $\begin{array}{l}\mathrm{P} \\
1\end{array}$ & $\begin{array}{l}\mathrm{P} \\
2\end{array}$ & $\begin{array}{l}\mathrm{P} \\
6\end{array}$ & $\begin{array}{l}\mathrm{P} \\
7\end{array}$ & $\begin{array}{l}\mathrm{P} \\
8\end{array}$ & $\begin{array}{l}\mathrm{P} \\
9\end{array}$ & $\begin{array}{c}\mathrm{P} \\
13\end{array}$ & $\begin{array}{c}\mathrm{P} \\
17\end{array}$ & $\begin{array}{c}\mathrm{P} \\
20\end{array}$ & $\begin{array}{c}\mathrm{P} \\
21\end{array}$ & $\begin{array}{c}\mathrm{P} \\
22\end{array}$ & $\begin{array}{c}\mathrm{P} \\
23\end{array}$ & $\begin{array}{c}\mathrm{P} \\
24\end{array}$ & $\begin{array}{c}\mathrm{P} \\
25 \\
\end{array}$ & $\begin{array}{c}\mathrm{P} \\
26 \\
\end{array}$ & $\begin{array}{c}P \\
27\end{array}$ & $\begin{array}{c}\mathbf{P} \\
28\end{array}$ & $\begin{array}{c}P \\
29 \\
\end{array}$ & $\begin{array}{c}\mathrm{P} \\
30\end{array}$ & $\begin{array}{c}\mathrm{P} \\
31\end{array}$ & $\begin{array}{c}\mathrm{P} \\
32\end{array}$ & $\begin{array}{c}\mathrm{P} \\
33\end{array}$ & $\begin{array}{c}\mathrm{P} \\
34\end{array}$ & $\begin{array}{c}\mathrm{P} \\
35\end{array}$ & $\begin{array}{c}\mathrm{P} \\
36\end{array}$ & $\begin{array}{c}\mathrm{P} \\
37\end{array}$ & $\mathrm{x}$ & tot \\
\hline \multirow{4}{*}{1} & a. equivoci & & & & & & & & & & & & & & & & & & & & & & & & & & & & \\
\hline & b. difetti & & & & & & & & & & & & & & & & & & & & & & & & & & & & \\
\hline & c. errori & & & & & & & & & & & & & & & & & & & & & & & & & & & & \\
\hline & d. peccati & & & & & & & & & & & & & & & & & & & & & & & & & & & & \\
\hline \multirow{4}{*}{2} & a. emergenza & & & & & & & & & & & & & & & & & & & & & & & & & & & & \\
\hline & b. imprevisto & & & & & & & & & & & & & & & & & & & & & & & & & & & 1 & \\
\hline & c. eventualità & & & & & & & & & & & & & & & & & & & & & & & & & & & 2 & \\
\hline & d. urgenza & & & & & & & & & & & & & & & & & & & & & & & & & & & 1 & 4 \\
\hline \multirow{4}{*}{3} & a. illimitato & & & & & & & & & & & & & & & & & & & & & & & & & & & 1 & \\
\hline & b. completo & & & & & & & & & & & & & & & & & & & & & & & & & & & 1 & \\
\hline & $\begin{array}{l}\text { c } \\
\text { incondizionato }\end{array}$ & & & & & & & & & & & & & & & & & & & & & & & & & & & 1 & \\
\hline & d. assoluto & & & & & & & & & & & & & & & & & & & & & & & & & & & & 3 \\
\hline \multirow{4}{*}{4} & a. oratori & & & & & & & & & & & & & & & & & & & & & & & & & & & 12 & \\
\hline & b. parlanti & & & & & & & & & & & & & & & & & & & & & & & & & & & & \\
\hline & c. parolai & & & & & & & & & & & & & & & & & & & & & & & & & & & & \\
\hline & d. relatori & & & & & & & & & & & & & & & & & & & & & & & & & & & 5 & 17 \\
\hline \multirow{4}{*}{5} & a. perizia & & & & & & & & & & & & & & & & & & & & & & & & & & & & \\
\hline & $\begin{array}{l}\text { b } \\
\text { considerazione }\end{array}$ & & & & & & & & & & & & & & & & & & & & & & & & & & & 1 & \\
\hline & c. stima & & & & & & & & & & & & & & & & & & & & & & & & & & & & \\
\hline & d. opinione & & & & & & & & & & & & & & & & & & & & & & & & & & & & 1 \\
\hline \multirow{4}{*}{6} & a. strumenti & & & & & & & & & & & & & & & & & & & & & & & & & & & & \\
\hline & b. arnesi & & & & & & & & & & & & & & & & & & & & & & & & & & & & \\
\hline & c. attrezzi & & & & & & & & & & & & & & & & & & & & & & & & & & & & \\
\hline & d. apparecchi & & & & & & & & & & & & & & & & & & & & & & & & & & & & \\
\hline \multirow{4}{*}{7} & a. diversità & & & & & & & & & & & & & & & & & & & & & & & & & & & 4 & \\
\hline & b. alterazioni & & & & & & & & & & & & & & & & & & & & & & & & & & & & \\
\hline & c. conversioni & & & & & & & & & & & & & & & & & & & & & & & & & & & & \\
\hline & d. varianti & & & & & & & & & & & & & & & & & & & & & & & & & & & & 4 \\
\hline
\end{tabular}




\begin{tabular}{|c|c|c|c|c|c|c|c|c|c|c|c|c|c|c|c|c|c|c|c|c|c|c|c|c|c|c|c|c|}
\hline & & $\begin{array}{l}\mathrm{P} \\
1\end{array}$ & $\begin{array}{l}\mathrm{P} \\
2\end{array}$ & $\begin{array}{l}\mathrm{P} \\
6 \\
\end{array}$ & $\begin{array}{l}\mathrm{P} \\
7\end{array}$ & $\begin{array}{l}\mathrm{P} \\
8 \\
\end{array}$ & $\begin{array}{l}\mathrm{P} \\
9\end{array}$ & $\begin{array}{c}\mathrm{P} \\
13 \\
\end{array}$ & $\begin{array}{c}\mathrm{P} \\
17\end{array}$ & $\begin{array}{c}\mathrm{P} \\
20\end{array}$ & $\begin{array}{c}\mathrm{P} \\
21\end{array}$ & \begin{tabular}{|c|c}
$\mathbf{P}$ \\
22
\end{tabular} & \begin{tabular}{|c|c|c}
$\mathrm{P}$ & 1 \\
23 & 2 \\
\end{tabular} & $\begin{array}{c}\mathrm{P} \\
24\end{array}$ & \begin{tabular}{c|c}
$\mathrm{P}$ & $\mathrm{I}$ \\
25 & 2 \\
\end{tabular} & \begin{tabular}{c|c}
$\mathrm{P}$ & \\
26 & 2 \\
\end{tabular} & \begin{tabular}{c|c}
$P$ & 1 \\
27 & 2 \\
\end{tabular} & \begin{tabular}{c|c}
$\mathbf{P}$ & $\mathrm{P}$ \\
28 & $2 \mathrm{C}$ \\
\end{tabular} & \begin{tabular}{l|l}
$\mathrm{P}$ & $\mathrm{F}$ \\
29 & 3 \\
\end{tabular} & & $\begin{array}{ll}P \\
1 & 32 \\
\end{array}$ & & $\begin{array}{c}\mathrm{P} \\
34 \\
\end{array}$ & $\begin{array}{c}\mathrm{P} \\
35 \\
\end{array}$ & \begin{tabular}{|c|l|} 
P & 1 \\
36 & 3 \\
\end{tabular} & \begin{tabular}{c|c}
$P$ & $\times$ \\
37 &
\end{tabular} & $\mathrm{x}$ & tot \\
\hline \multirow{4}{*}{8} & a. vocaboli & & & & & & & & & & & & & & & & & & & & & & & & & & & \\
\hline & b. argomenti & & & & & & & & & & & & & & & & & & & & & & & & & & & \\
\hline & c. segni & & & & & & & & & & & & & & & & & & & & & & & & & & & \\
\hline & d. significati & & & & & & & & & & & & & & & & & & & & & & & & & & & \\
\hline \multirow{4}{*}{9} & $\begin{array}{l}\text { a } \\
\text { concentrazione }\end{array}$ & & & & & & & & & & & & & & & & & & & & & & & & & & & \\
\hline & b. riflessione & & & & & & & & & & & & & & & & & & & & & & & & & & & \\
\hline & c. attenzione & & & & & & & & & & & & & & & & & & & & & & & & & & & \\
\hline & d. divergenza & & & & & & & & & & & & & & & & & & & & & & & & & & & \\
\hline \multirow{4}{*}{10} & a. espressione & & & & & & & & & & & & & & & & & & & & & & & & & & & \\
\hline & $\begin{array}{l}\mathrm{b} \\
\text { atteggiamento }\end{array}$ & & & & & & & & & & & & & & & & & & & & & & & & & & & \\
\hline & c. impostazione & & & & & & & & & & & & & & & & & & & & & & & & & & 2 & \\
\hline & d. esibizione & & & & & & & & & & & & & & & & & & & & & & & & & & & 2 \\
\hline \multirow{4}{*}{11} & a. dedicato & & & & & & & & & & & & & & & & & & & & & & & & & & 2 & \\
\hline & b. rivolto & & & & & & & & & & & & & & & & & & & & & & & & & & & \\
\hline & c. concentrato & & & & & & & & & & & & & & & & & & & & & & & & & & & \\
\hline & $\begin{array}{l}\mathrm{d} \\
\text { rappresentativo }\end{array}$ & & & & & & & & & & & & & & & & & & & & & & & & & & & 2 \\
\hline \multirow{4}{*}{12} & a. ripetitivo & & & & & & & & & & & & & & & & & & & & & & & & & & & \\
\hline & b. regolare & & & & & & & & & & & & & & & & & & & & & & & & & & 4 & \\
\hline & c. normale & & & & & & & & & & & & & & & & & & & & & & & & & & 1 & \\
\hline & d. ordinato & & & & & & & & & & & & & & & & & & & & & & & & & & 2 . & 7 \\
\hline \multirow{4}{*}{13} & a. merito & & & & & & & & & & & & & & & & & & & & & & & & & & & \\
\hline & b. rilievo & & & & & & & & & & & & & & & & & & & & & & & & & & 2 & \\
\hline & c. pregio & & & & & & & & & & & & & & & & & & & & & & & & & & & \\
\hline & d. valore & & & & & & & & & & & & & & & & & & & & & & & & & & & 2 \\
\hline \multirow{4}{*}{14} & a. aspetto & & & & & & & & & & & & & & & & & & & & & & & & & & & \\
\hline & b. esponente & & & & & & & & & & & & & & & & & & & & & & & & & & & \\
\hline & c. ingrediente & & & & & & & & & & & & & & & & & & & & & & & & & & & \\
\hline & d. elemento & & & & & & & & & & & & & & & & & & & & & & & & & & 2 & 2 \\
\hline \multirow{4}{*}{15} & a. episodio & & & & & & & & & & & & & & & & & & & & & & & & & & & \\
\hline & b. fenomeno & & & & & & & & & & & & & & & & & & & & & & & & & & & \\
\hline & c. affare & & & & & & & & & & & & & & & & & & & & & & & & & & & \\
\hline & d. problema & & & & & & & & & & & & & & & & & & & & & & & & & & & \\
\hline
\end{tabular}


Dall'indagine emerge il comportamento divergente di un numero considerevole di NE (30\%) in merito all'item \# 4.

A nostro parere vanno anche considerate, benché di impatto minore, le divergenze occorse durante la compilazione degli items \#\# 12, 13 e 14.

Vediamo in dettaglio ciascuno dei casi.

\subsubsection{Item \# 4}

Riportiamo il testo completato con evidenziata la chiave dell'item \# 4.

Come parliamo? Come sta l'italiano oggi? Come sta cambiando? È vero che chi parla male pensa male e vive male, come diceva Nanni Moretti? L'Università Ca' Foscari di Venezia lancia dati (0) allarmanti : all'ultimo esame di ammissione alla Facoltà di Lettere il 44 per cento dei candidati sarebbero stati bocciati per (1) errori ortografici.

Ma i linguisti ridimensionano l' (2) emergenza e alcuni luoghi comuni, come quello che vede in internet, tv e inglese il male (3) assoluto.

"La lingua italiana e la sua grammatica stanno benone" afferma il professor Tullio De Mauro, ex Ministro dell'Istruzione: "Mai nei secoli erano state adoperate da un numero così ampio di (4) parlanti e mai tanto studiate da stranieri che apprendono l'italiano come seconda lingua". Siamo noi che stiamo meno bene: secondo una (5) stima soltanto un terzo della popolazione ha gli (6) strumenti culturali sufficienti a servirsi con scioltezza e correttezza di una lingua piena di (7) varianti come l'italiano".

4. A) oratori B) paranti C) parolai D) relatori

La chiave è (b) parlanti; i distrattori maggiormente selezionati dai NE sono:

- (a) oratori $(29 \%)$

- (d) relatori $(13 \%)$

Il fatto che, nel complesso, oltre il $40 \%$ dei NE abbia ritenuto plausibile l'inserzione dei distrattori (a) e (d) induce a interrogarci sulla plausibilità delle opzioni.

Se inseriamo il distrattore (a), il testo, a nostro parere, risulta comunque dotato di senso: 
"La lingua italiana e la sua grammatica stanno benone" afferma il professor Tullio De Mauro, ex Ministro dell'Istruzione: "Mai nei secoli erano state adoperate da un numero così ampio di (4) oratori e mai tanto studiate da stranieri che apprendono l'italiano come seconda lingua". Siamo noi che stiamo meno bene: secondo una (5) stima soltanto un terzo della popolazione ha gli (6) strumenti culturali sufficienti a servirsi con scioltezza e correttezza di una lingua piena di (7) varianti come l'italiano".

Lo stesso dicasi se inseriamo il distrattore (d):

"La lingua italiana e la sua grammatica stanno benone" afferma il professor Tullio De Mauro, ex Ministro dell'Istruzione: "Mai nei secoli erano state adoperate da un numero così ampio di (4) relatori e mai tanto studiate da stranieri che apprendono l'italiano come seconda lingua". Siamo noi che stiamo meno bene: secondo una (5) stima soltanto un terzo della popolazione ha gli (6) strumenti culturali sufficienti a servirsi con scioltezza e correttezza di una lingua piena di (7) varianti come l'italiano".

Nel brano emerge un'opposizione tra un ampio numero di soggetti che usa l'italiano e studia/ conosce la grammatica e chi invece la conosce meno.

A conoscerla meno sono gli italiani ("siamo noi"): due terzi della popolazione non paiono avere un vocabolario ampio né essere corretti.

A usarla in modo appropriato e corretto e a conoscerla approfonditamente sono piuttosto gli stranieri: il termine "parlanti" vale appunto a includere nel cerchio della comunità italofona tutti coloro che ricorrono alla lingua di Dante, anche se non sono cittadini italiani e risiedono fuori dai confini nazionali.

Per un lettore non è, però, illogico pensare - con un'interpretazione suggerita dai distrattori (a) e (d) - che un gran numero di professionisti della comunicazione (dell'arte retorica, i.e. oratori, o conferenzieri, i.e. relatori) vantino un'ottima padronanza della lingua (in opposizione a un numero minore nel passato) e che, per loro tramite, in aggiunta al contributo del gran numero di studenti che l'apprendono, la lingua italiana si mantenga viva e solida. 


\subsubsection{Item \# 12}

All'item \# 12 si legge

Il linguaggio era più didascalico, anche perché (11) rivolto ad un pubblico meno alfabetizzato, mentre ora è diventato più (12) ripetitivo e meno creativo.

12. A) ripetitivo $\mathrm{B}$ ) regolare $\mathrm{C}$ ) normale $\mathrm{D}$ ) ordinato

Il $10 \%$ dei NE ha optato per il distrattore (b) *regolare.

Il linguaggio era più didascalico, anche perché (11) rivolto ad un pubblico meno alfabetizzato, mentre ora è diventato più (12) * regolare e meno creativo.

C'è da notare che il certificatore ha espunto l'aggettivo presente nella fonte autentica, "formulaico", e inserito come chiave "ripetitivo", appunto.

L'intenzione del giornalista, nell'usare l'aggettivo "formulaico" in oppozione a "didascalico", era quella di definire come l'italiano si sia evoluto verso una forma meno impostata, meno ricca, per essere caratterizzato da formule ricorrenti, e trasmesso con un registro più colloquiale (basti mettere a confronto il parlato di un presentatore televisivo di oggi con quello di uno degli anni '60 per rendersene conto).

Il certificatore ha pensato, a ragione, che "ripetitivo" sia equivalente a "formulaico".

Vero è che "regolare", il distrattore (b), all'infuori dalla microlingua dell'informatica, non rappresenta una collocazione cui si ricorra. Perché allora un numero discreto di NE vi ha fatto ricorso? La risposta pare risiedere in un uso figurato della lingua, tal per cui un "linguaggio regolare" può prefigurarsi come forma di comunicazione priva di vezzi, ordinaria, alla portata di tutti, che rasenta il colloquiale.

\subsubsection{Item \# 13}

All'item \# 13 si legge:

Si abusa di slogan o frasi fatte: valzer delle poltrone, guerra dei numeri... Scorciatoie comunicative che diventano subito modelli perdendo il (13) valore metaforico.

13. A) merito $B$ ) rilievo C) pregio $D$ ) valore 
Un paio di NE ha optato per il distrattore (b), "rilievo", anziché per la chiave (d) "valore". Il termine "valore" ha diversi significati, tra i quali, in questo contesto, secondo l'intenzione dell'autore del TO (cfr. Dizionario Sabatini Coletti), quello di: "significato, funzione, soprattutto in ambito linguistico (es.: 'l'aggettivo ha assunto qui v. di sostantivo')".

"Valore", però, sta anche per "importanza", "significatività": il certificatore ha scelto i tre distrattori in quanto condividono questa seconda, più ampia, accezione - estranea, lo ripetiamo, rispetto all'intenzione dell'autore del TO.

Ora, mentre "merito", il distrattore (a), ha una connotazione personale (si tratta del risultato di un'azione degna di onore) e non si adatta alla frase in questione, "rilievo" e "pregio" possono essere inseriti a ragione nel testo. Il senso dell'enunciato cambia, certo, ma è pur sempre logicamente possibile. Il testo, in tal caso, ci informerebbe, cioè, che un processo di lessicalizzazione ha portato alla costituzione di unità autonome, come per esempio "valzer di poltrone", nelle quali il pregio dell'origine figurata è venuto meno; si è opacizzata cioè quella caratteristica distintiva (i.e. rilievo) dell'espressione che è la sua natura analogica.

\subsubsection{Item \# 14}

All'item \# 14 si legge:

Stefano Bartezzaghi, direttore del recente Festival della Linguistica a Poggibonsi (Siena), cerca l'(14) aspetto positivo: "L'italiano viene calpestato di più perché si parla di più, e questo non è un (15) fenomeno negativo. Più auto circolano e più incidenti, statisticamente, si contano".

14. A) aspetto $B$ ) esponente C) ingrediente D) elemento

"Aspetto positivo" costituisce una collocazione ricorrente. Per quanto "elemento" sia inserito come opzione non corretta, ci pare sia possibile intenderlo, in questo caso, come corrispettivo di "aspetto", in quanto a "elemento di una serie", i.e. tratto di una totalità. Esempi attinti dalla rete possono convincere, a tal proposito: "Hotel Residence Pompeo: il principale elemento positivo è la spiaggia" (www.tripadvisor.it); "L'unico elemento positivo è la vicinanza a piedi" (www.tripadvisor.it).

\subsubsection{Cloze B}

Di seguito presentiamo testo e item del cloze B (C2, sessione estiva CILS 2012)

Completa il testo. Scegli una delle proposte di completamento (elencate sotto). 
UN LIBRO PER (0) DEGLI SMS

capire

COME CAMBIA LA LINGUA ITALIANA AI TEMPI

Se telefonando ti scrivo è il titolo del libro appena pubblicato dall' Accademia della Crusca che spiega il progressivo (1) dell'italiano al telefono, dal parlato al digitato. "Quando si scrivono sms, l'obiettivo di chi scrive è quello di (2) immediatamente con il messaggio il proprio

(3) e quindi essere veloce", spiega la presidente dell'Accademia, Nicoletta Maraschio.

"La scrittura in questo caso ha un (4) effimero, tanto da essere definita scrittura volatile, cioè che se ne va".

Ma allora, come si può difendere l'italiano da queste nuove forme di comunicazione? "Dobbiamo evitare", afferma la presidente, "che ci sia un (5) verso la scrittura normale che noi usiamo nelle (6) di questo tipo di comunicazione effimera scritto. Per questo è importante che la scuola intervenga non tanto censurando il modo di scrivere gli sms, perché non servirebbe, ma (7) la capacità dei ragazzi di muoversi all'interno della lingua senza contaminare le caratteristiche di una varietà con un'altra varietà".

Come farlo? "La scuola oggi", sostiene Nicoletta Maraschio, "ha il compito di (8) linguisticamente i propri allievi nella loro lingua materna, sviluppando la competenza comunicativa come capacità di variare la lingua nelle diverse situazioni del parlato e dello scritto".

"Ma il problema", prosegue, "non riguarda solo l'italiano. Abbiamo realizzato questo libro, Se telefonando ti scrivo, insieme ad un (9) polacco e a un altro belga: anche loro hanno rilevato le stesse forme di (10) dell'italiano". nelle loro lingue che sono grosso modo (11) a quelle

"È molto caratteristico di questo tipo di scritture effimere", prosegue la presidente, "il fatto che lo scrivente non rilegge i testi, (12) degli apostrofi diverso o (13) tanto quello della (14) in molti casi errori di ortografia o uso degli accenti o

"La lettura", conclude Nicoletta Maraschio, "è fondamentale e soprattutto oggi, perché la lettura significa lentezza, significa riflessione, capacità di (15) un testo e apprezzarlo. La lettura è quindi uno strumento fondamentale".

\begin{tabular}{lllll}
\hline 0. & A) capire & B) intendere & C) sentire & D) calcolare \\
\hline 1. & A) fiorire & B) perfezionarsi & C) maturare & D) evolvere \\
\hline 2. & A) cogliere & B) toccare & C) raggiungere & D) conquistare \\
\hline 3. & A) conversatore & B) interlocutore & C) compagno & D) corrispondente \\
\hline 4. & A) modo & B) tratto & C) segno & D) carattere \\
\hline 5. & A) cambio & B) trasloco & C) versamento & D) travaso \\
\hline
\end{tabular}




\begin{tabular}{lllll}
\hline 6. & A) possibilità & B) cause & C) occasioni & D) offerte \\
\hline 7. & A) tonificando & B) risanando & C) alzando & D) incrementando \\
\hline 8 & A) addestrare & B) formare & C) modellare & D) configurare \\
\hline 9. & A) collega & B) socio & C) complice & D) compagno \\
\hline 10. & A) diminuzione & B) riduzione & C) restrizione & D) abbreviazione \\
\hline 11. & A) uniformi & B) congruenti & C) analoghe & D) conformi \\
\hline 12. & A) facendo & B) indicando & C) decidendo & D) fissando \\
\hline 13. & A) eccezionale & B) anomalo & C) anormale & D) raro \\
\hline 14. & A) regolarità & B) puntualità & C) correttezza & D) scrupolosità \\
\hline 15. & A) imparare & B) percepire & C) sapere & D) comprendere \\
\hline
\end{tabular}

Nella tabella che segue abbiamo raccolto le sole risposte errate $(n=21)$.

Legenda

in grigio sono riportate le chiavi

$\mathrm{x}$ : errori per ogni singolo distrattore

in rosso sono riportate le risposte divergenti da parte dei NE

$\mathrm{y}$ : risposte mancanti

tot: errori per ogni singolo item 


\begin{tabular}{|c|c|c|c|c|c|c|c|c|c|c|c|c|c|c|c|c|c|c|c|c|c|c|c|c|c|}
\hline & & $\begin{array}{l}\mathrm{P} \\
2\end{array}$ & $\begin{array}{l}P \\
3\end{array}$ & $\begin{array}{l}\mathrm{P} \\
4\end{array}$ & $\begin{array}{l}\mathrm{P} \\
7\end{array}$ & $\begin{array}{l}\mathrm{P} \\
8\end{array}$ & $\begin{array}{l}\mathrm{P} \\
9\end{array}$ & \begin{tabular}{c|c}
$\mathrm{P}$ & \\
14 & 1
\end{tabular} & \begin{tabular}{c|c}
$\mathrm{P}$ & $\mathrm{F}$ \\
15 & 1
\end{tabular} & \begin{tabular}{c|c}
$\mathrm{P}$ & \\
16 & 1 \\
\end{tabular} & $\begin{array}{c}P \\
17\end{array}$ & $\begin{array}{c}\mathrm{P} \\
18\end{array}$ & $\begin{array}{c}\mathrm{P} \\
19\end{array}$ & $\begin{array}{l}P \\
21\end{array}$ & $\begin{array}{c}\mathrm{P} \\
23\end{array}$ & $\begin{array}{c}\mathrm{P} \\
26\end{array}$ & $\begin{array}{c}\mathrm{P} \\
27\end{array}$ & $\begin{array}{c}\mathrm{P} \\
28\end{array}$ & $\begin{array}{c}\mathrm{P} \\
29\end{array}$ & $\begin{array}{c}\mathrm{P} \\
30\end{array}$ & $\begin{array}{c}\mathrm{P} \\
35\end{array}$ & $\begin{array}{c}\mathrm{P} \\
36\end{array}$ & $\mathrm{x}$ & $\mathrm{y}$ & tot \\
\hline \multirow{4}{*}{1} & a. fiorire & & & & & & & & & & & & & & & & & & & & & & 2 & & \\
\hline & b. perfezionarsi & & & & & & & & & & & & & & & & & & & & & & 1 & & \\
\hline & c. maturare & & & & & & & & & & & & & & & & & & & & & & & & \\
\hline & d. evolvere & & & & & & & & & & & & & & & & & & & & & & & & 3 \\
\hline \multirow{4}{*}{2} & a. cogliere & & & & & & & & & & & & & & & & & & & & & & & & \\
\hline & b. toccare & & & & & & & & & & & & & & & & & & & & & & & & \\
\hline & c. raggiungere & & & & & & & & & & & & & & & & & & & & & & & & \\
\hline & d. conquistare & & & & & & & & & & & & & & & & & & & & & & & & \\
\hline \multirow{4}{*}{3} & a. conversatore & & & & & & & & & & & & & & & & & & & & & & & & \\
\hline & b. interlocutore & & & & & & & & & & & & & & & & & & & & & & & & \\
\hline & c. compagno & & & & & & & & & & & & & & & & & & & & & & & & \\
\hline & d. corrispondente & & & & & & & & & & & & & & & & & & & & & & & & \\
\hline \multirow{4}{*}{4} & a. modo & & & & & & & & & & & & & & & & & & & & & & & & \\
\hline & b. tratto & & & & & & & & & & & & & & & & & & & & & & & & \\
\hline & c. segno & & & & & & & & & & & & & & & & & & & & & & 1 & & \\
\hline & d. carattere & & & & & & & & & & & & & & & & & & & & & & & & 1 \\
\hline \multirow{4}{*}{5} & a. cambio & & & & & & & & & & & & & & & & & & & & & & 10 & & \\
\hline & b. trasloco & & & & & & & & & & & & & & & & & & & & & & 1 & & \\
\hline & c. versamento & & & & & & & & & & & & & & & & & & & & & & 1 & & \\
\hline & d. travaso & & & & & & & & & & (?) & & & & & & & & & & & & & 1 & 13 \\
\hline \multirow{4}{*}{6} & a. possibilità & & & & & & & & & & & & & & & & & & & & & & & & \\
\hline & b. cause & & & & & & & & & & & & & & & & & & & & & & & & \\
\hline & c. occasioni & & & & & & & & & & & & & & & & & & & & & & & & \\
\hline & d. offerte & & & & & & & & & & & & & & & & & & & & & & & & \\
\hline \multirow{6}{*}{7} & a. tonificando & & & & & & & & & & & & & & & & & & & & & & 1 & & \\
\hline & b. risanando & & & & & & & & & & & & & & & & & & & & & & & & \\
\hline & c. alzando & & & & & & & & & & & & & & & & & & & & & & 2 & & \\
\hline & d. incrementando & & & & & & & & & & & & & & & & & & & & & & & & 4 \\
\hline & & $\mathrm{P}$ & $\mathrm{P}$ & $\mathrm{P}$ & $\mathrm{P}$ & $\mathrm{P}$ & $\mathrm{P}$ & $\mathrm{P}$ & $\mathrm{P}$ & $\mathrm{P}$ & $\mathrm{P}$ & $\mathrm{P}$ & $\mathrm{P}$ & $\mathrm{P}$ & $\mathrm{P}$ & $\mathrm{P}$ & $\mathrm{P}$ & $\mathrm{P}$ & $\mathrm{P}$ & $\mathrm{P}$ & $\mathrm{P}$ & $\mathrm{P}$ & $\mathrm{x}$ & $\mathrm{y}$ & tot \\
\hline & & 2 & 3 & 4 & 7 & 8 & 9 & 14 & 15 & 16 & 17 & 18 & 19 & 21 & 23 & 26 & 27 & 28 & 29 & 30 & 35 & 36 & & & \\
\hline
\end{tabular}




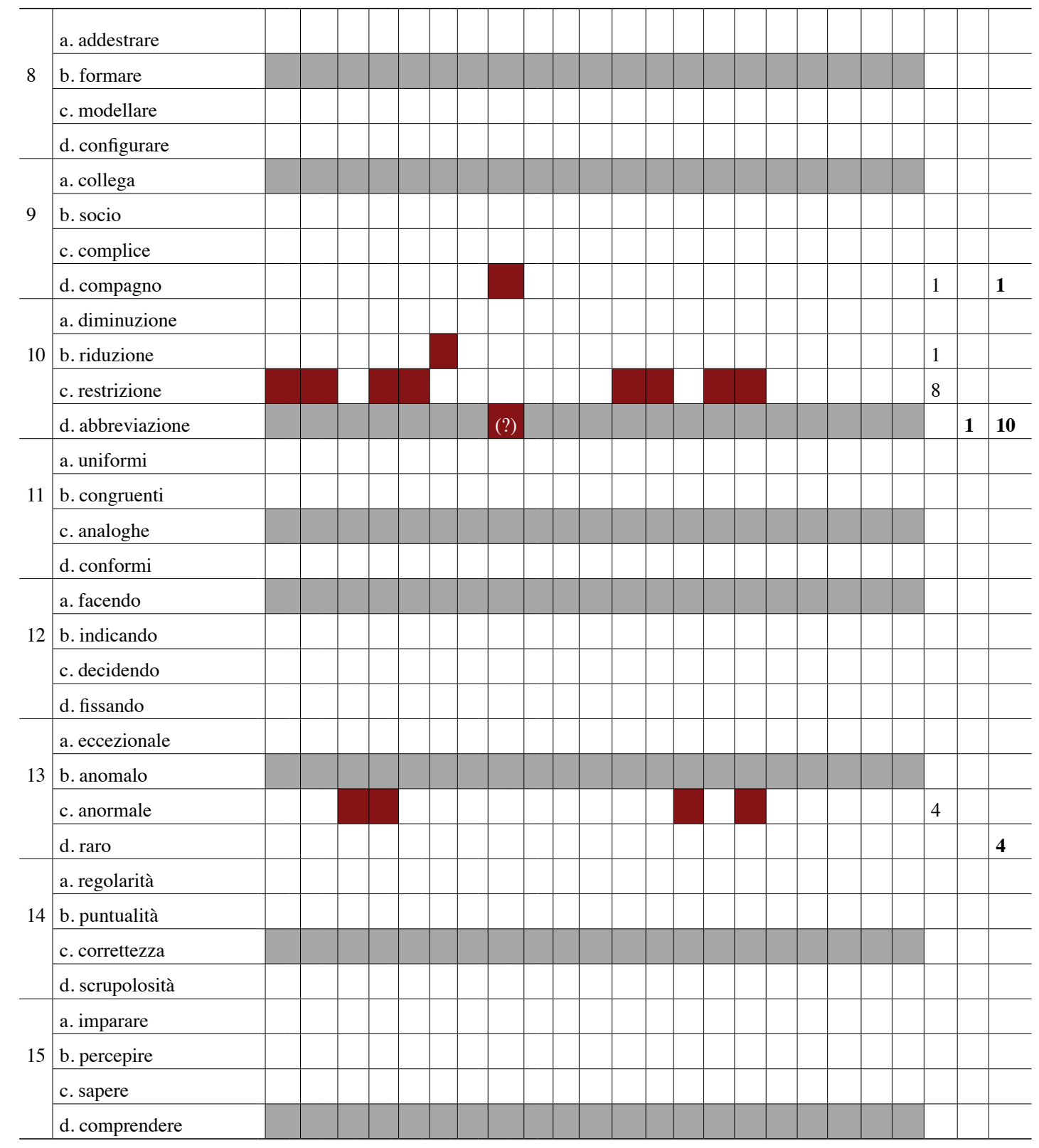


Nei paragrafi a seguire ci soffermiamo sugli item \#\# 5 e 10, in corrispondenza ai quali un nutrito numero di NE ha optato per un distrattore.

\subsubsection{Item \# 5}

Riportiamo il testo completato e la chiave evidenziata dell'item \# 5:

"Quando si scrivono sms, l'obiettivo di chi scrive è quello di (2) raggiungere immediatamente con il messaggio il proprio (3) interlocutore e quindi essere veloce", spiega la presidente dell'Accademia, Nicoletta Maraschio. "La scrittura in questo caso ha un (4) carattere effimero, tanto da essere definita scrittura volatile, cioè che se ne va".

Ma allora, come si può difendere l'italiano da queste nuove forme di comunicazione? "Dobbiamo evitare", afferma la presidente, "che ci sia un (5) travaso di questo tipo di comunicazione effimera verso la scrittura normale che noi usiamo nelle (6) occasioni in cui dobbiamo utilizzare l'italiano scritto.

\section{A) cambio B) trasloco C) versamento D) travaso}

L'item si è rivelato difficile per molti; un volontario ha addirittura omesso la risposta. Il distrattore che ha riscosso parecchi consensi è stato (a) "cambio".

A differenza dei casi precedenti, la scelta divergente non ci pare legittima: non è la "comunicazione effimera" soggetta a un cambio (verso quale termine sarebbe il cambio?), piuttosto è la scrittura, costretta, per effetto della contaminazione ("travaso", appunto) della prima, a modificarsi.

Rimane da chiedersi come mai molti NE abbiano errato. Al momento l'unica ragione plausibile pare risiedere in una lettura poco accurata. Una scelta più istintiva che ragionata, vien da dire; una NE ha, per esempio, sacrificato la semantica allo stile con cognizione di causa: "sapevo che «travaso» era l'opzione corretta, ma non mi piaceva, e ho messo «cambio»".

Ad ogni modo, benché la scelta del gruppo sia impropria, c'è da chiedersi se, nell'ottica di rimodellare il test, non sia opportuno comunque operare una modifica all'item. Vogliamo cioè dire che, se un item risulta problematico per un numero considerevole di NE, è opinabile lo si possa somministrare a studenti di livello avanzato, nonostante la scelta dell'item writer sia fuori discussione. 


\subsubsection{Item \# 10}

Riportiamo testo completo e chiave evidenziata dell'item \# 10:

"Ma il problema", prosegue, "non riguarda solo l'italiano. Abbiamo realizzato questo libro, Se telefonando ti scrivo, insieme ad un (9) collega polacco e a un altro belga: anche loro hanno rilevato le stesse forme di (10) abbreviazione nelle loro lingue che sono grosso modo (11) analoghe a quelle dell'italiano".

0 . A) diminuzione $\mathrm{B}$ ) riduzione $\mathrm{C}$ ) restrizione $\mathrm{D}$ ) abbreviazione

Il testo oggetto di analisi riguarda, abbiamo visto, la lingua degli sms, "comunicazione effimera", in opposizione a una lingua più accurata e completa, qual è la lingua scritta in generale.

La lingua degli sms, in effetti, è stringata, funziona per acronomi e troncamenti; è popolata, come recita la chiave (d), da "forme di abbreviazione" (così, nel TM; da notare che nel TO la specificazione è al plurale: "forme di abbreviazioni”).

Un fitto gruppo di NE ritiene però plausibile l'inserzione del distrattore (c) "restrizione".

Tale opzione è, a nostro avviso, plausibile, in quanto riferibile alla lingua come codice: ciò di cui si parla (la "comunicazione effimera" degli sms) sarebbe, in tal senso, caratterizzato dal fatto di essere un codice "ristretto", rispetto alla lingua più estesa, più ricca, qual è quella scritta in generale. Si rilegga il passaggio, in tal senso:

Ma il problema", prosegue, "non riguarda solo l'italiano. Abbiamo realizzato questo libro, Se telefonando ti scrivo, insieme ad un (9) collega polacco e a un altro belga: anche loro hanno rilevato le stesse forme di (10) riduzione nelle loro lingue che sono grosso modo (11) analoghe a quelle dell'italiano.

\section{Conclusioni}

Attraverso questo saggio ribadiamo l'importanza di una validazione esterna rispetto a quella che può avvenire dal pool degli item writer o da campioni di studenti.

In Alderson, Clapham e Wall si legge:

It is therefore absolutely crucial in all test development, for whatever purpose, at whatever level of learner ability and however trivial the consequences of failure 
on test might be, that some person or persons other than the individual item writer look closely at each item, respond to the item as a student would, reflect upon what abilities are required for successful completion of the item/task, and then compare what he or she thinks the item is testing with what the item writer claims it tests. (1995, p. 63)

La certificazione CILS prevede un rigoroso protocollo a garanzia dell'affidabilità e della validità delle prove:

Allo scopo di garantire l'affidabilità delle prove il Centro CILS ha adottato delle procedure per la garanzia dell'affidabilità delle prove che si attuano in due fasi: prima e dopo la somministrazione del test. Per le prove chiuse si ricorre all'item analysis [...]. Dopo la prima stesura delle prove viene realizzato un pre-testing per verificare la validità e l'affidabilità degli item. Il pre-testing è effettuato su un campione di individui con competenze adeguate al livello per cui la prova è costruita. Le risposte ottenute sono sottoposte ad analisi statistiche, per rilevare gli item troppo facili o troppo difficili; per individuare se ci sono distrattori, cioè le risposte non esatte proposte accanto alle risposte esatte nelle prove oggettive, che hanno una percentuale di scelta troppo bassa o troppo alta; per verificare se gli item sono in grado di discriminare i candidati più competenti da quelli meno competenti, e per poter effettuare le opportune tarature delle prove.

Dopo la somministrazione del test, l'analisi degli item viene ripetuta sulle risposte date dai candidati che hanno effettivamente sostenuto l'esame CILS. (BARNI et al., 2009, p. 19)

La questione è che, a nostro avviso, ciò non basta. Va data risposta all'appello di Alderson, Clapham e Wall, precisando l'opportunità di coinvolgere anche persone non legate al testing. Il giudizio dei NE può costituire, abbiamo visto, un utile parametro di riferimento. Anche nel caso in cui un nutrito gruppo di NE erri o si dimostri titubante, dovrebbe valere la regola di rimodellare il test. 


\section{Riferimenti bibliografici}

ALDERSON, C.; CLAPHAM, C.; WALL, D. Language Test Construction and Evaluation. Cambridge: CUP, 1995.

BARNI, M. et al. Linee guida CILS. Certificazione di italiano come LS Università per stranieri di Siena. Perugia: Guerra, 2009.

CAI, H. Partial Dictation as a Measure of EFL Listening Proficiency: Evidence from Confirmatory Factor Analysis. In Language Testing, 27: 1-23, 2012.

CONSIGLIO D'EUROPA. Quadro comune europeo di riferimento per le lingue: apprendimento, insegnamento, valutazione (QCER). Firenze: La Nuova Italia, 2002.

HULSTIJN, J. H. Language Proficiency in Native and Non-native Speakers: An Agenda for Research and Suggestions for Second-language Assessment. In Language Assessment Quarterly, 8(3): 229-249, 2011.

MULDER, K.; HULSTIJN, J. H. Linguistic Skills of Adult Native Speakers, as A Function of Age and Level of Education. In Applied Linguistics, 32, 5: 475-494, 2011.

\section{Riferimenti sitografici}

UNIVERSITÀ PER STRANIERI DI SIENA. CENTRO CILS. Analisi delle strutture di comunicazione - Prova n.3. In Livello CILS QUATTRO - C2, Giugno 2009.

www.gedi.it/cils/file/89/200/file/c2-q(3).pdfAccesso: 14.02.2015.

UNIVERSITÀ PER STRANIERI DI SIENA. CENTRO CILS. Analisi delle strutture di comunicazione - Prova n.3. In Livello CILS QUATTRO - C2, Giugno 2012.

http://cils.unistrasi.it/public/articoli/200/Files/quaderno_quattro-c2_giugno_2012.pdfAccesso: 14.02.2015. TRIPADVISOR ITALIA.

http://www.tripadvisor.it/ Accesso: 14.02.2015.

\section{Testi autentici su cui sono state basate le prove}

COME CAMBIA LA LINGUA ITALIANA AI TEMPI DEGLI SMS. In La Stampa, 5 gennaio 2012. http://www.lastampa.it/2012/01/05/cultura/come-cambia-la-lingua-italianaai-tempi-degli-smstAxKczqsGQ6jglVptTZRDN/pagina.html Accesso: 15/06/2015

MARRESE, E. Grammatica: viene avanti uno strano italiano. Colpa di internet e TV? No, nostra. In Venerdì di Repubblica: 26-29, 10 aprile 2009. 


\section{Appendice 1. Cloze A: modifiche incorse sul TO}

Il brano su cui è stato ricavato il cloze è frutto di una manipolazione di un brano autentico.

Nella presentazione affiancata di TO e TM, a seguire, modifiche e criticità sono segnalate come segue.

testo originale

rosso: parte soggetta a espunzione

giallo: parte soggetta a una manipolazione significativa

PTO: problemi nel testo originale

TESTO MODIFICATO CILS

celeste: inserzioni

PTM: problemi nel testo modificato

Nel testo modificato le chiavi sono contraddistinte da numero e riportate in grassetto 
TESTO ORIGINALE

Riportato su:

http://blog.italia150.it/wp-content/uploads/2009/04/grammatica.pdf

Ó Emilio Marrese, Venerdì di Repubblica 10/4/2009 (p. 26-29) testo modificato CILS

(con chiavi inserite)

\section{GRAMMATICA: VIENE AVANTI UNO STRANO ITALIANO. COLPA DI INTERNET E TV? NO, NOSTRA}

[...]

Come parliamo? Come sta l'italiano oggi? Come sta cambiando? "In questo momento sono sette le atlete che possono fare sua la maratona". "I due giocatori si evalgono". Giusto un paio di recenti perle da telecronache Rai. I soliti sportivi, si dirà. Ma non è che altrove la nostra lingua se la passi meglio. Chi parla male, pensa male e vive male, come diceva Moretti? È allarmante che, all'ultimo esame di ammissione alla Facoltà di Lettere dell'Università veneziana Ca' Foscari, il 44 per cento dei candidati $[$ PTO1 $\rightarrow$ ] siano stati bocciati per gli errori ortografici?

I linguisti ridimensionano l'emergenza e alcuni luoghi comuni, come quello che vede in internet, tv e inglese il male assoluto. "La lingua italiana e la sua grammatica stanno benone" afferma il professor Tullio De Mauro, ex Ministro dell'Istruzione: "Mai nei secoli erano state adoperate da un numero così ampio di parlanti e mai tanto studiate da stranieri che apprendono l'italiano come seconda lingua. Siamo noi che stiamo meno bene: secondo una stima soltanto un terzo della popolazione ha gli strumenti culturali sufficienti a servirsi con scioltezza e correttezza di una lingua piena di varianti come l'italiano".

Negli ultimi dieci anni, secondo una ricerca Treccani (questa è vera), i giornali hanno introdotto o in qualche modo lanciato 4.163 neologismi, accettati nei vocabolari: da gossipare ad attapirarsi, da botulinata a fannullonismo. "Una volta l'informazione parlava meglio" sostiene Andrea De Benedetti, giornalista e linguista [PTO2 $\rightarrow$ ] autore del libro Val più la pratica. Prima grammatica immorale della lingua italiana. "C'era più attenzione per la scrittura e la pronuncia, si facevano corsi di dizione. Ora la provenienza geografica nell'inflessione è quasi ostentata. Il linguaggio era più didascalico, anche perché rivolto ad un pubblico meno alfabetizzato, mentre ora è diventato più formulare e meno creativo. $\mathrm{Si}$ abusa di slogan o frasi fatte: valzer delle poltrone, guerra dei numeri... Scorciatoie comunicative che diventano subito cliché perdendo il valore metaforico. C'è più sciatteria”. Stefano Bartezzaghi, direttore del recente Festival della Linguistica a Poggibonsi (Siena), in programma venerdì e sabato prossimi, cerca l'aspetto positivo: "L'italiano viene calpestato di più perché si parla di più, e questo non è un fenomeno negativo. Più auto circolano e più incidenti, statisticamente, si contano". [...]

\section{GRAMMATICA: VIENE AVANTI UNO STRANO ITALIANO. COLPA DI INTERNET E TV? NO, NOSTRA}

Come parliamo? Come sta l'italiano oggi? Come sta cambiando? È vero che chi parla male pensa male e vive male, come diceva Nanni Moretti? L'Università Ca' Foscari di Venezia lancia dati (0) allarmanti: all'ultimo esame di ammissione alla Facoltà di Lettere il 44 per cento dei candidati $[$ PTM1 $\rightarrow$ ] sarebbero stati bocciati per (1) errori ortografici.

Ma i linguisti ridimensionano l' (2) emergenza e alcuni luoghi comuni, come quello che vede in internet, tv e inglese il male (3) assoluto. "La lingua italiana e la sua grammatica stanno benone" afferma il professor Tullio De Mauro, ex Ministro dell'Istruzione: "Mai nei secoli erano state adoperate da un numero così ampio di (4) parlanti e mai tanto studiate da stranieri che apprendono l'italiano come seconda lingua. Siamo noi che stiamo meno bene: secondo una (5) stima soltanto un terzo della popolazione ha gli (6) strumenti culturali sufficienti a servirsi con scioltezza e correttezza di una lingua piena di (7) varianti come l'italiano".

Negli ultimi dieci anni, secondo una ricerca Treccani, i giornali hanno introdotto o in qualche modo lanciato 4.163 (8) vocaboli nuovi: da gossipare ad attapirarsi, da botulinata a fannullonismo. "Una volta l'informazione parlava meglio" sostiene Andrea De Benedetti, giornalista e linguista. [PTM2 $\rightarrow$ ] C'era più (9) attenzione per la scrittura e la pronuncia, si facevano corsi di dizione. Ora la provenienza geografica nell' (10) espressione è quasi ostentata. Il linguaggio era più didascalico, anche perché (11) rivolto ad un pubblico meno alfabetizzato, mentre ora è diventato più (12) ripetitivo e meno creativo. Si abusa di slogan o frasi fatte: valzer delle poltrone, guerra dei numeri... Scorciatoie comunicative che diventano subito modelli perdendo il (13) valore metaforico. C'è più sciatteria". Stefano Bartezzaghi, direttore del recente Festival della Linguistica a Poggibonsi (Siena), cerca l'(14) aspetto positivo: "L'italiano viene calpestato di più perché si parla di più, e questo non è un (15) fenomeno negativo. Più auto circolano e più incidenti, statisticamente, si contano". 
A seguire riportiamo, in maniera dettagliata, ciascuna criticità.

\begin{tabular}{|c|c|c|c|}
\hline & TESTO ORIGINALE & EMENDAMENTO SUGGERITO & COMMENTO \\
\hline PTO1 & $\begin{array}{l}\text { È allarmante che, all'ultimo esame } \\
\text { di ammissione alla Facoltà di Lettere } \\
\text { dell'Università veneziana Ca' Foscari, } \\
\text { il } 44 \text { per cento dei candidati }[\rightarrow] \text { siano } \\
\text { stati bocciati per gli errori ortografici? }\end{array}$ & $\begin{array}{l}\text { È allarmante che, all'ultimo esame } \\
\text { di ammissione alla Facoltà di Lettere } \\
\text { dell'Università veneziana Ca' Foscari, } \\
\text { il } 44 \text { per cento dei candidati }[\rightarrow] \text { sia } \\
\text { stato bocciato per gli errori ortografici? }\end{array}$ & $\begin{array}{l}\text { L'accordo non } \\
\text { è corretto. }\end{array}$ \\
\hline PTO2 & $\begin{array}{l}\text { "Una volta l'informazione parlava } \\
\text { meglio" sostiene Andrea De De } \\
\text { Benedetti, giornalista e linguista }[\rightarrow] \\
\text { autore del libro Val più la pratica. } \\
\text { Prima grammatica immorale della } \\
\text { lingua italiana. }\end{array}$ & $\begin{array}{l}\text { "Una volta l'informazione parlava } \\
\text { meglio" sostiene Andrea De Benedetti, } \\
\text { giornalista e linguista }[\rightarrow] \text {, autore } \\
\text { del libro Val più la pratica. Prima } \\
\text { grammatica immorale della lingua } \\
\text { italiana. }\end{array}$ & $\begin{array}{l}\text { È necessario } \\
\text { apporre una } \\
\text { virgola a fine } \\
\text { inciso. }\end{array}$ \\
\hline
\end{tabular}

\begin{tabular}{|c|c|c|c|}
\hline & TESTO MODIFICATO CILS & EMENDAMENTO SUGGERITO & COMMENTO \\
\hline PTM1 & $\begin{array}{l}\text { È allarmante che, all'ultimo esame } \\
\text { di ammissione alla Facoltà di Lettere } \\
\text { dell'Università veneziana Ca' Foscari, } \\
\text { il } 44 \text { per cento dei candidati }[\rightarrow] \\
\text { sarebbero stati bocciati per errori } \\
\text { ortografici? }\end{array}$ & $\begin{array}{l}\text { È allarmante che, all'ultimo esame } \\
\text { di ammissione alla Facoltà di Lettere } \\
\text { dell'Università veneziana Ca' Foscari, il } \\
44 \text { per cento dei candidati }[\rightarrow \text { ] sia stato } \\
\text { bocciato per gli errori ortografici? }\end{array}$ & $\begin{array}{l}\text { L'accordo } \\
\text { non è corretto } \\
\text { (come in } \\
\text { PTO1). }\end{array}$ \\
\hline PTM2 & $\begin{array}{l}\text { "Una volta l'informazione parlava } \\
\text { meglio" sostiene Andrea De Benedetti, } \\
\text { giornalista e linguista. }[\rightarrow \text { ] C'era } \\
\text { più attenzione per la scrittura e } \\
\text { la pronuncia, si facevano corsi di } \\
\text { dizione. Ora la provenienza geografica } \\
\text { nell'espressione è quasi ostentata. Il } \\
\text { linguaggio era più didascalico, anche } \\
\text { perché rivolto ad un pubblico meno } \\
\text { alfabetizzato, mentre ora è diventato } \\
\text { più ripetitivo e meno creativo. Si } \\
\text { abusa di slogan o frasi fatte: valzer } \\
\text { delle poltrone, guerra dei numeri... } \\
\text { Scorciatoie comunicative che } \\
\text { diventano subito modelli perdendo il } \\
\text { valore metaforico. C'è più sciatteria". }\end{array}$ & $\begin{array}{l}\text { "Una volta l'informazione parlava } \\
\text { meglio" sostiene Andrea De Benedetti, } \\
\text { giornalista e linguista. [ } \rightarrow \text { ] "C'era più } \\
\text { attenzione per la scrittura e la pronuncia, } \\
\text { si facevano corsi di dizione. Ora la } \\
\text { provenienza geografica nell'espressione } \\
\text { è quasi ostentata. Il linguaggio era più } \\
\text { didascalico, anche perché rivolto ad un } \\
\text { pubblico meno alfabetizzato, mentre } \\
\text { ora è diventato più ripetitivo e meno } \\
\text { creativo. Si abusa di slogan o frasi } \\
\text { fatte: valzer delle poltrone, guerra dei } \\
\text { numeri... Scorciatoie comunicative che } \\
\text { diventano subito modelli perdendo il } \\
\text { valore metaforico. C'è più sciatteria". }\end{array}$ & $\begin{array}{l}\text { È necessario } \\
\text { aprire le } \\
\text { virgolette, } \\
\text { altrimenti } \\
\text { non si sa da } \\
\text { dove cominci } \\
\text { il discorso } \\
\text { diretto. }\end{array}$ \\
\hline
\end{tabular}




\section{Appendice 2. Cloze B: modifiche incorse sul TO}

Anche in questo caso il certificatore ha operato delle modifiche su un articolo di giornale. Nella presentazione affiancata di TO e TM, modifiche e criticità sono segnalate come segue.

testo originale

rosso: parte soggetta a espunzione

giallo: parte soggetta a una manipolazione significativa

PTO: problemi nel testo originale

TESTO MODIFICATO CILS

celeste: inserzioni

PTM: problemi nel testo modificato

Nel testo modificato le chiavi sono contraddistinte da numero e riportate in grassetto. 


\section{Come cambia la lingua italiana ai tempi degli sms}

La presidente dell' Accademia della Crusca 'L'obiettivo non è tanto quello della correttezza quanto quello di una comunicatività immediata" ROMA

"Se telefonando ti scrivo" è il titolo del [PTO1 $\rightarrow$ ] appena pubblicato dall'Accademia della Crusca che spiega l'evolvere dell'italiano al telefono, dal parlato al digitato. Ig «Quando si scrivono sms oppure email l'obiettivo di chi scrive è quello di arrivare immediatamente con il messaggio al proprio interlocutore e quindi essere veloce - spiega la presidente dell'Accademia, Nicoletta Maraschio - la scrittura in questo caso ha un carattere effimero, tanto da essere definita scrittura $[\mathbf{P T O 2} \rightarrow$ ] volatili, cioè che se ne va». Ma allora, come si può difendere l'italiano da queste nuove forme? «Il problema secondo la presidente - è che non ci sia un travaso di questo tipo di comunicazione effimera verso la scrittura normale che noi usiamo tutti i giorni per scrivere relazioni oppure a scuola o nelle occasioni in cui dobbiamo utilizzare l'italiano scritto. Per questo è importante che la scuola intervenga non tanto censurando, perché non servirebbe, ma alzando la capacità dei ragazzi di muoversi all'interno di una lingua complessa e variegata come è la nostra oggi e come sono in genere tutte le lingue. L'importante è sapersi muovere attraverso le tante varietà e non mescolare tra loro. Non far quindi debordare le caratteristiche di una varietà in un'altra varietà». Come farlo? «Insegnando - rimarca la presidente dell'Accademia della Crusca - È molto importante intanto parlarne con i ragazzi. La scuola di oggi ha un compito essenziale: quello di formare linguisticamente i propri allievi nella propria lingua materna. Formare vuol dire appunto dare una competenza comunicativa, cioè la capacità di variare la lingua a seconda delle diverse situazioni comunicative, quindi nel parlato e nei diversi tipi di scritto. Per questo è necessario iniziare a farlo da subito, dalle elementari fino alla fine del percorso scolastico». «Ma il problema - prosegue - non riguarda solo l'italiano, ma anche le altre lingue. Abbiamo realizzato questo libro "Se telefonando ti scrivo" insieme ad un collega polacco e un altro belga, anche loro hanno rilevato le stesse forme di abbreviazione nelle loro lingue che sono grosso modo analoghe a quelle dell'italiano». «È molto caratteristico di questo tipo di scritture effimere - prosegue - il fatto che lo scrivente non rilegge $\mathrm{i}$ testi, determinando in molti casi errori di ortografie o parole scritte unite là dove dovrebbero essere separate o uso degli accenti o degli apostrofi diverso o anomalo, quindi agrammaticale, perché l'obiettivo appunto non è tanto quello della correttezza quanto quello di una comunicatività immediata». «La lettura - conclude Nicoletta Maraschio - è fondamentale e soprattutto oggi, perché la lettura significa "lentezza". È importante che in un mondo così veloce come quello di oggi si abituino i giovani alla lentezza che significa riflessione, capacità di acquisire un testo e rileggerlo e apprezzarlo. La lettura è quindi uno strumento fondamentale». Eppure, contrazioni, abbreviazioni di intere frasi che non sono nuove alla nostra lingua, soprattutto scritta. Infatti, come spiega la presidente della Crusca, «chi studia i manoscritti medievali sa che questo tipo di abbreviazione si applicavano già allora per un problema di costi della carta e quindi di massima utilizzazione di ogni spazio». «Tanto - continua - che oggi abbiamo difficoltà a tradurre gli scritti medievali, per questo ci sono dei manuali che ci aiutano a farlo. Esattamente come online si trovano manuali che ci servono a spiegare molte delle abbreviazioni che sono in circolazione negli sms. Questo vuol dire che si è formata una specie di tradizione nell'uso di alcune abbreviazioni».
Un libro per (0) capire come cambia la lingua italiana ai tempi degli sms

Se telefonando ti scrivo è il titolo del libro appena pubblicato dall'Accademia della Crusca che spiega il progressivo (1) evolvere dell'italiano al telefono, dal parlato al digitato. "Quando si scrivono sms, l'obiettivo di chi scrive è quello di (2) raggiungere immediatamente con il messaggio il proprio (3) interlocutore e quindi essere veloce", spiega la presidente dell'Accademia, Nicoletta Maraschio. "La scrittura in questo caso ha un (4) carattere effimero, tanto da essere definita scrittura volatile, cioè che se ne va".

Ma allora, come si può difendere l'italiano da queste nuove forme di comunicazione? "Dobbiamo evitare", afferma la presidente, "che ci sia un (5) travaso di questo tipo di comunicazione effimera verso la scrittura normale che noi usiamo nelle (6) occasioni in cui dobbiamo utilizzare l'italiano scritto. Per questo è importante che la scuola intervenga non tanto censurando il modo di scrivere gli sms, perché non servirebbe, ma (7) incrementando la capacità dei ragazzi di muoversi all'interno della lingua [PTM1 $\rightarrow$ ] senza contaminare le caratteristiche di una varietà con un'altra varietà".

Come farlo? "La scuola oggi", sostiene Nicoletta Maraschio, "ha il compito di (8) formare linguisticamente i propri allievi nella loro lingua materna, sviluppando la competenza comunicativa come capacità di variare la lingua nelle diverse situazioni del parlato e dello scritto".

"Ma il problema", prosegue, "non riguarda solo l'italiano. Abbiamo realizzato questo libro, Se telefonando ti scrivo, insieme ad un (9) collega polacco e a un altro belga: anche loro hanno rilevato le stesse forme di (10) abbreviazioni nelle loro lingue che sono grosso modo (11) analoghe a quelle dell'italiano".

"È molto caratteristico di questo tipo di scritture effimere", prosegue la presidente, "il fatto che lo scrivente non rilegge i testi, (12) facendo in molti casi errori di ortografia o uso degli accenti o degli apostrofi diverso o (13) anomalo, quindi agrammaticale, perché l'obiettivo appunto non è tanto quello della (14) correttezza quanto quello di una comunicatività immediata".

"La lettura [PTM2 $\rightarrow$ ], conclude Nicoletta Maraschio, è fondamentale e soprattutto oggi, perché la lettura significa lentezza, significa riflessione, capacità di (15) comprendere un testo e apprezzarlo. La lettura è quindi uno strumento fondamentale". 
A seguire riportiamo, in maniera dettagliata, ciascuna criticità.

\begin{tabular}{|c|c|c|c|}
\hline & TESTO ORIGINALE & EMENDAMENTO SUGGERITO & COMMENTO \\
\hline PTO1 & $\begin{array}{l}\text { "Se telefonando ti scrivo" è il } \\
\text { titolo del }[\rightarrow \text { ] appena pubblicato } \\
\text { dall'Accademia della Crusca che } \\
\text { spiega l'evolvere dell'italiano al } \\
\text { telefono, dal parlato al digitato. Ig }\end{array}$ & $\begin{array}{l}\text { "Se telefonando ti scrivo" è } \\
\text { il titolo del }[\rightarrow] \text { libro appena } \\
\text { pubblicato dall'Accademia della } \\
\text { Crusca che spiega l'evolvere } \\
\text { dell'italiano al telefono, dal parlato } \\
\text { al digitato. }\end{array}$ & $\begin{array}{l}\text { Manca } \\
\text { un'informazione }\end{array}$ \\
\hline \multirow[t]{2}{*}{ PTO2 } & $\begin{array}{l}\text { tanto da essere definita scrittura }[\rightarrow] \\
\text { volatili }\end{array}$ & $\begin{array}{l}\text { tanto da essere definita scrittura } \\
{[\rightarrow] \text { volatile }}\end{array}$ & $\begin{array}{l}\text { Manca l'accordo } \\
\text { sostantivo- } \\
\text { aggettivo }\end{array}$ \\
\hline & TESTO MODIFICATO CILS & EMENDAMENTO SUGGERITO & COMMENTO \\
\hline PTM1 & $\begin{array}{l}\text { incrementando la capacità dei } \\
\text { ragazzi di muoversi all'interno della } \\
\text { lingua }[\rightarrow] \text { senza contaminare le } \\
\text { caratteristiche di una varietà con } \\
\text { un'altra varietà". }\end{array}$ & $\begin{array}{l}\text { incrementando la capacità dei } \\
\text { ragazzi di muoversi all'interno } \\
\text { della lingua }[\rightarrow] \text {, senza } \\
\text { contaminare le caratteristiche di } \\
\text { una varietà con un'altra varietà". }\end{array}$ & $\begin{array}{l}\text { È necessario } \\
\text { l'inserimento di } \\
\text { una virgola }\end{array}$ \\
\hline PTM2 & $\begin{array}{l}\text { "La lettura }[\rightarrow] \text {, conclude Nicoletta } \\
\text { Maraschio, è fondamentale e } \\
\text { soprattutto oggi, perché la lettura } \\
\text { significa lentezza, significa } \\
\text { riflessione, capacità di (15) } \\
\text { comprendere un testo e apprezzarlo. } \\
\text { La lettura è quindi uno strumento } \\
\text { fondamentale". }\end{array}$ & $\begin{array}{l}\text { "La lettura }[\rightarrow] \text { ", conclude } \\
\text { Nicoletta Maraschio, }[\rightarrow] \text { " è } \\
\text { fondamentale e soprattutto oggi, } \\
\text { perché la lettura significa lentezza, } \\
\text { significa riflessione, capacità di } \\
(15) \text { comprendere un testo e } \\
\text { apprezzarlo. La lettura è quindi } \\
\text { uno strumento fondamentale". }\end{array}$ & $\begin{array}{l}\text { Mancano le } \\
\text { virgolette di } \\
\text { apertura e } \\
\text { chiusura }\end{array}$ \\
\hline
\end{tabular}

